\title{
Inversion Mutation Abnormality
}

National Cancer Institute

\section{Source}

National Cancer Institute. Inversion Mutation Abnormality. NCI Thesaurus. Code C45589.

A structural change in genomic DNA where the 5' to $3^{\prime}$ order of a nucleotide sequence is completely reversed to the $3^{\prime}$ to $5^{\prime}$ order relative to its adjacent sequences. This inversion is termed either pericentric, if it includes the centromere of a chromosome, or pancentric, if it excludes the centromere. An inversion mutation abnormality may be heritable or occur somatically. 